\title{
La prise en compte des incertitudes dans l'estimation du coût des dommages dus aux inondations
}

\author{
Including uncertainties while estimating damage cost due to flood \\ par Eric Gaume, Gilles Hubert \\ ENPC - CEREVE
}

Jean-Philippe Torterotot

Direction départementale de l'agriculture et de la forêt de l'Isère

Expected damage cost reduction estimations due to flood control reservoirs on the river Loire are presented in this paper. The estimated damage costs are the direct damages to housing (buildings and their content), and direct and indirect damages to activities including the agricultural activity. After a short description of the method used and the results obtained, a particular focus is put on the evaluation and analysis of the uncertainties in the damage cost estimates.

A sensitivity analysis shows that refinements in the description of the land use in the flooded area or in the relations between flooding characteristics and elementary loss costs do not guaranty a more precise damage cost estimation. Moreover, the spreading of the mean annual cost distributions obtained is mainly influenced by the uncertainties in the flood return periods. Nevertheless these results depend on the estimation method used and on the case study, and must be confirmed by other studies of the same type.

\section{INTRODUCTION}

Les tentatives d'évaluation du coût des dommages dus aux inondations, qu'il s'agisse de bilans économiques de crues réelles ou d'études prospectives sur des crues à venir, sont peu nombreuses [1-8]. Les évaluations économiques sont par exemple pratiquement absentes des études de vulnérabilité réalisées dans le cadre des plans de prévention des risques. Par ailleurs, le recours à des études économiques préalables à la réalisation d'ouvrages de protection contre les crues n'est pas systématique en France. Dans d'autres pays européens comme la Grande-Bretagne, l'évaluation des bénéfices économiques des aménagements envisagés est obligatoire, et des guides méthodologiques $[16,17]$ destinés aux maîtres d'ouvrage et aux bureaux d'étude ont été rédigés.

Plusieurs explications peuvent être avancées : la complexité des approches économiques, le travail important de collecte d'information qu'elles nécessitent et par voie de conséquence leur coût supposé. L'expérience montre cependant que les études économiques sont relativement peu coûteuses si on les compare à d'autres types d'études usuellement réalisées sur les inondations : le coût du volet économique d'une étude sur les crues de la Seine et leurs dommages potentiels en région Ile-de-France récemment réalisée par l'Institution des grands lacs de Seine [8] représentait moins du quart du coût des volets hydrologique et hydraulique.
Les résultats de ces études, souvent réduits à leur plus simple expression (montant total des dommages relatifs à une crue ou coût moyen annuel des dommages sur un tronçon de cours d'eau) peuvent paraître décevants, d'autant plus que ces estimations sont entachées de fortes incertitudes. Mais le montant total des dommages est loin d'être le seul résultat des études économiques. L'identification des différents désordres (destructions matérielles, coupures de réseaux), le poids relatif des dommages par type d'enjeu (habitat, activités industrielles et commerciales, agriculture, bâtiments et équipements publics), la localisation des secteurs particulièrement exposés, l'inventaire des équipements sensibles, sont autant d'éléments de connaissances des conséquences des crues sur une vallée. Ils permettront de sensibiliser les acteurs locaux et éventuellement mettront en évidence la nécessité ou non de réaliser des équipements de protection, aideront à définir des priorités d'action et à repenser l'organisation des secours.

Enfin, il n'existe pas d'étude standard du coût des dommages dus aux inondations. Le type d'enjeux et de désordres auxquels on cherche à attribuer une valeur monétaire dépend des questions auxquelles le maître d'ouvrage souhaite répondre. Ces études peuvent se limiter au chiffrage des destructions matérielles ou bien aux coûts des désordres induits localement par l'inondation (opérations de secours, interruptions et ralentissement des activités pendant et après la crue, 
coupures de réseaux d'alimentation en eau, électricité, gaz, et de réseaux de transport...) ou à une échelle géographique plus grande (conséquences sur les fournisseurs et clients de l'interruption de certaines activités, marché de reconstruction). Le coût des dommages estimé peut être une simple somme des dommages supportés par chaque agent ou bien les montants restant à la charge des agents après indemnisation par les assurances et éventuellement intervention des collectivités territoriales et de l'Etat. Enfin, les études économiques peuvent se limiter à l'estimation des dommages au moment de la crue ou s'intéresser aux conséquences à long terme de ces dommages : fragilisation du tissu économique et éventuellement faillite de certaines entreprises, endettement des agents économiques [5], conséquences sur les finances des collectivités territoriales. Cela explique que la plupart des analyses économiques des dommages liés aux inondations ont fait l'objet d'études de définition [1,7,8].

Il reste que malgré les difficultés de leur mise en œuvre, les études économiques apportent, tout comme les études hydrologique et hydraulique bien plus répandues, des connaissances sur les inondations dont on ne saurait se priver.

Cet article vise, au travers d'une étude de cas, à illustrer les grands principes de l'évaluation des dommages dus aux inondations et à révéler les difficultés et les limites de son application. L'utilisation d'un modèle (CIFLUPEDE [4]) permet d'automatiser les calculs des coûts de dommages directs aux habitations et aux activités industrielles, artisanales, commerciales et agricoles ainsi que les dommages indirects liés à l'interruption de ces activités. Les calculs sont effectués pour des crues de différentes périodes de retour, ce qui permet d'évaluer un coût moyen annuel des dommages. La constitution d'une importante base de données regroupant des informations hydrologiques, hydrauliques, d'occupation des sols, et économiques a permis d'appliquer ce modèle sur 245 secteurs de vallées inondables représentant près de 2000 kilomètres de linéaires de cours d'eau du bassin de la Loire.

Le principal intérêt du modèle CIFLUPEDE réside dans la simplification des algorithmes de calcul, en particulier pour la partie hydraulique du modèle. La rapidité des calculs permet, grâce à des simulations répétées de type Monte-Carlo, d'évaluer l'effet des incertitudes sur les données utilisées dans le modèle : aléa hydrologique, fonctionnement hydraulique des cours d'eau, seuils (seuils d'apparition des dommages, seuils de rupture de digues...) ou encore données économiques (valeurs des biens exposés, fonctions d'endommagement). L'application de CIFLUPEDE présentée dans cet article vise à évaluer la réduction du coût des dommages potentiels sur la Loire moyenne du fait de la réalisation d'un nouveau barrage. Les principaux objectifs de ce travail étaient les suivants :

- Adapter un modèle existant conçu pour la vallée de la Loire en situation actuelle pour y intégrer les effets d'aménagements structurels, en l'occurrence d'un barrage projeté.

- Simuler et analyser les incertitudes sur les valeurs estimées de coût des dommages, en tenant compte de la cohérence des sources entre les situations influencées et non influencées par le barrage.

- Identifier les principales sources d'incertitudes sur lesquelles devront éventuellement porter des efforts complémentaires de collecte de données.

Cette étude est la seule à notre connaissance à tenter d'estimer les incertitudes sur les montants des dommages dus aux

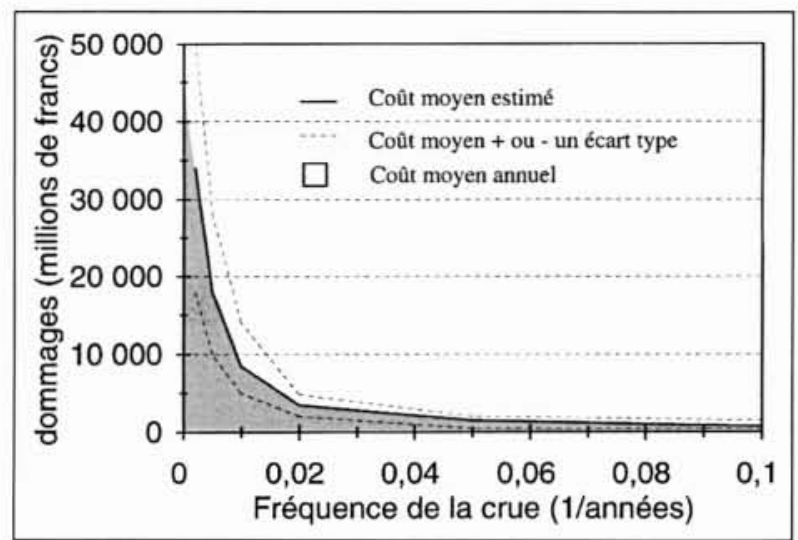

1. Exemple de résultats de calcul de coût des dommages pour des crues de fréquences variées et calcul du coût moyen annuel. Cas de la Loire moyenne.

inondations. Les résultats présentés illustrent la dispersion importante des estimations de coût des dommages et mettent en évidence l'irréductibilité de certaines sources d'incertitudes. De ce fait, les décisions concernant la réalisation d'aménagements de protection contre les inondations ne peuvent se baser uniquement sur les résultats des évaluations économiques.

\section{D PRINCIPES D'ESTIMATION DU COÛT DES DOMMAGES DANS LE MODÈLE CIFLUPEDE}

L'unité de base sur laquelle est effectué le calcul des dommages est le secteur géographique. Il s'agit d'une zone de la plaine inondable homogène du point de vue de l'occupation des sols et soumise à un processus d'inondation unique : débordement, remous aval, rupture de digue suivant les cas.

Les lois de probabilité des débits de pointe de crue, à partir desquels seront calculés les surfaces submergées et les hauteurs et durées de submersion, sont définies à l'échelle du bief de rivière (unité hydrologique et hydraulique). Chaque bief est rattaché à une station hydrométrique. Un bief comporte en général plusieurs secteurs.

Le calcul du coût des dommages est effectué pour des crues de référence. Il s'agit i) des crues de période de retour $1,2,3,4,5,10,20,50,100,200$ et 500 ans, ii) des crues correspondant à des seuils de processus hydrauliques (débordement, rupture de digue...), iii) et des crues correspondant aux seuils d'apparition des dommages et de submersion complète de chaque secteur.

L'ensemble des résultats obtenus permet de reconstituer la courbe d'évolution du coût des dommages en fonction de la fréquence de la crue pour chacun des secteurs, et de calculer par intégration le coût moyen annuel : espérance mathématique du coût annuel des dommages (cf. figure 1).

L'estimation du coût des dommages compte quatre étapes successives dans le modèle CIFLUPEDE. Le processus de calcul est schématisé dans la figure 2.

(1) L'étape hydrologique permet de définir le débit de pointe de crue (Qi) et la hauteur d'eau maximale atteinte $(\mathrm{Hi})$ à la station hydrométrique pour chacune des crues de référence. Inversement elle permet d'estimer les périodes de retour (Ti) des crues correspondant à des seuils sur chacun des secteurs, ces seuils étant généralement définis en hauteur d'eau au niveau de la station hydrométrique. 


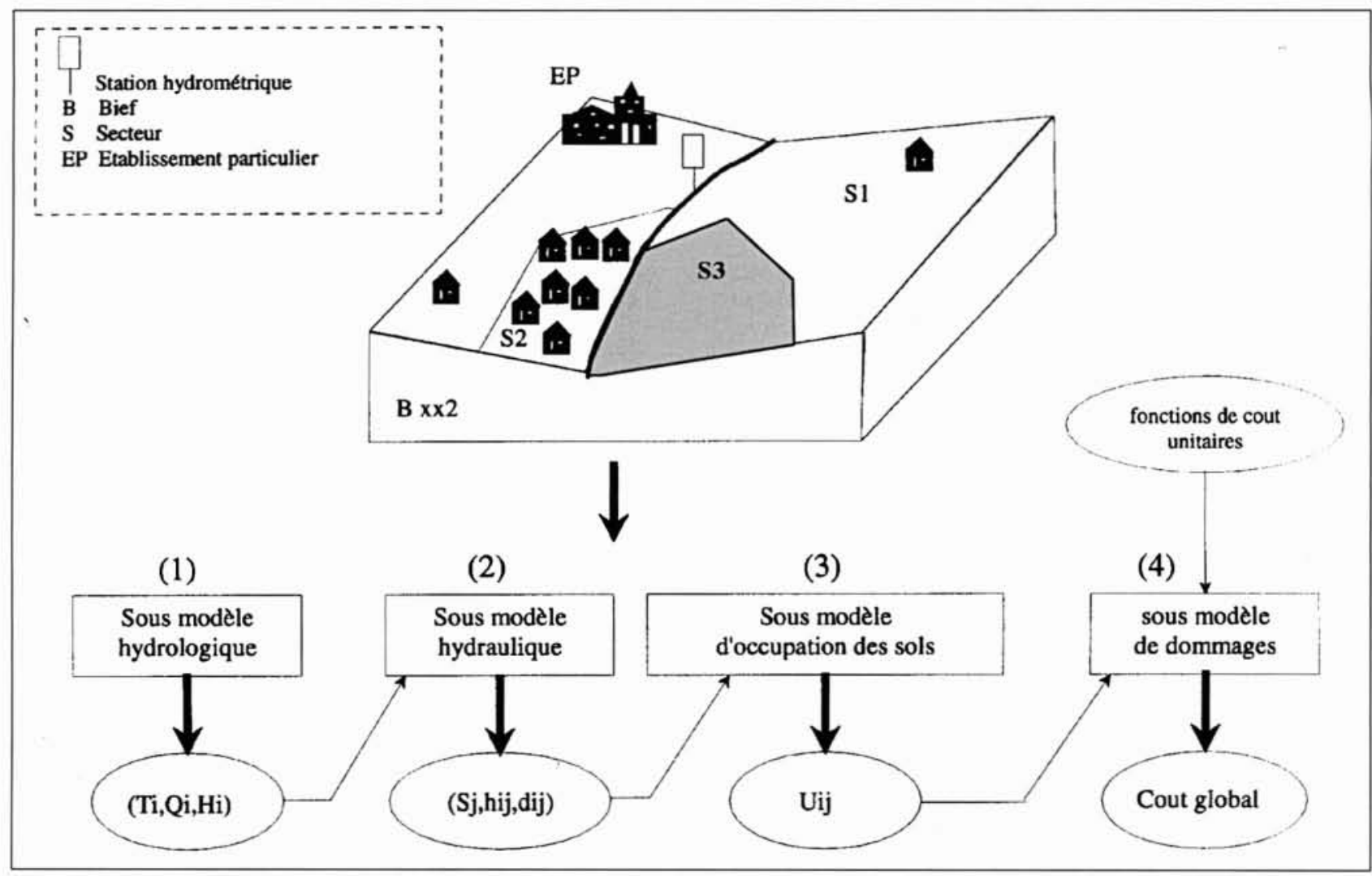

2. Schématisation de la procédure de calcul du coût des dommages dans CIFLUPEDE

(2) La surface $(\mathrm{Sj})$ inondée sur chacun des secteurs, ainsi que la distribution des hauteurs (hij) et des durées (dij) de submersion sont calculées au cours de l'étape hydraulique. Cette surface est obtenue par interpolation ou extrapolation de la courbe "hauteur ou débit de pointe mesuré à la station hydrométrique versus surface inondée". Cette courbe est établie pour chaque secteur à partir de relevés de crues historiques ou de résultats de simulations hydrauliques. L'interpolation tient compte de la forme de la vallée et du mécanisme de submersion. Pour les secteurs concernés par la rupture de digue, deux scénarios sont envisagés - rupture ou non rupture - chacun étant affecté d'une probabilité dépendant de la hauteur de crue (Hi).

(3) Les informations obtenues à l'étape précédente sont ensuite croisées avec les données d'occupation des sols de chaque secteur. On obtient pour chaque crue de référence et chaque secteur, les caractéristiques de l'inondation $(\mathrm{Sj}$, hij, dij), et le pourcentage de la surface inondée que représente chaque type d'occupation des sols (Uij).

(4) L'utilisation de fonctions de coûts unitaires dépendant des caractéristiques d'inondation et du type d'occupation des sols, permet d'établir pour chaque crue de référence le coût global des dommages par secteur et par bief. Les fonctions de coûts utilisées proviennent pour partie de la littérature [13-15], et pour partie d'enquêtes réalisées auprès de particuliers et d'industriels sur des zones ayant subi des inondations.

\section{III $\square$ PRISE EN COMPTE DES INCERTI- TUDES DANS LE MODÈLE}

Les incertitudes sont l'un des éléments importants d'une prise de décision : elles peuvent conduire soit à modifier la décision par rapport à une situation déterministe, soit à repousser la décision dans l'attente d'éléments d'information complémentaires. Cette question se pose d'autant plus dans le cas du modèle CIFLUPEDE, que celui-ci est basé sur des représentations parfois très simplifiées de la réalité, et que les données d'entrée peuvent être entachées d'erreurs non négligeables.

Les sources d'incertitudes sont multiples dans le modèle. Les principales concernent :

- l'aléa hydrologique : courbe de tarage, ajustement d'une loi de probabilité théorique, variabilité d'échantillonnage, hauteur d'eau au droit du secteur en fonction du débit de pointe de crue.

- la définition des crues par une composante unique (débit de pointe, hauteur) à laquelle on peut affecter une période de retour, et à partir de laquelle sont déduites les autres caractéristiques de la crue : surface submergée, durée de submersion. - les différents seuils des processus pris en compte : seuil d'apparition des dommages, seuils de débordement, de rupture de digue.

- la représentation simplifiée de l'occupation des sols, et de la topographie du lit majeur.

- la méconnaissance de la valeur des biens exposés.

- les fonctions de coûts unitaires.

A ces sources viennent s'ajouter les incertitudes concernant l'effet du barrage-réservoir :

- réduction du débit de pointe de crue pour une période de retour donnée.

- modification de la forme de l'hydrogramme de crue et donc de la durée de submersion correspondant à un débit de pointe.

Le modèle CIFLUPEDE, malgré les nombreuses simplifi- 


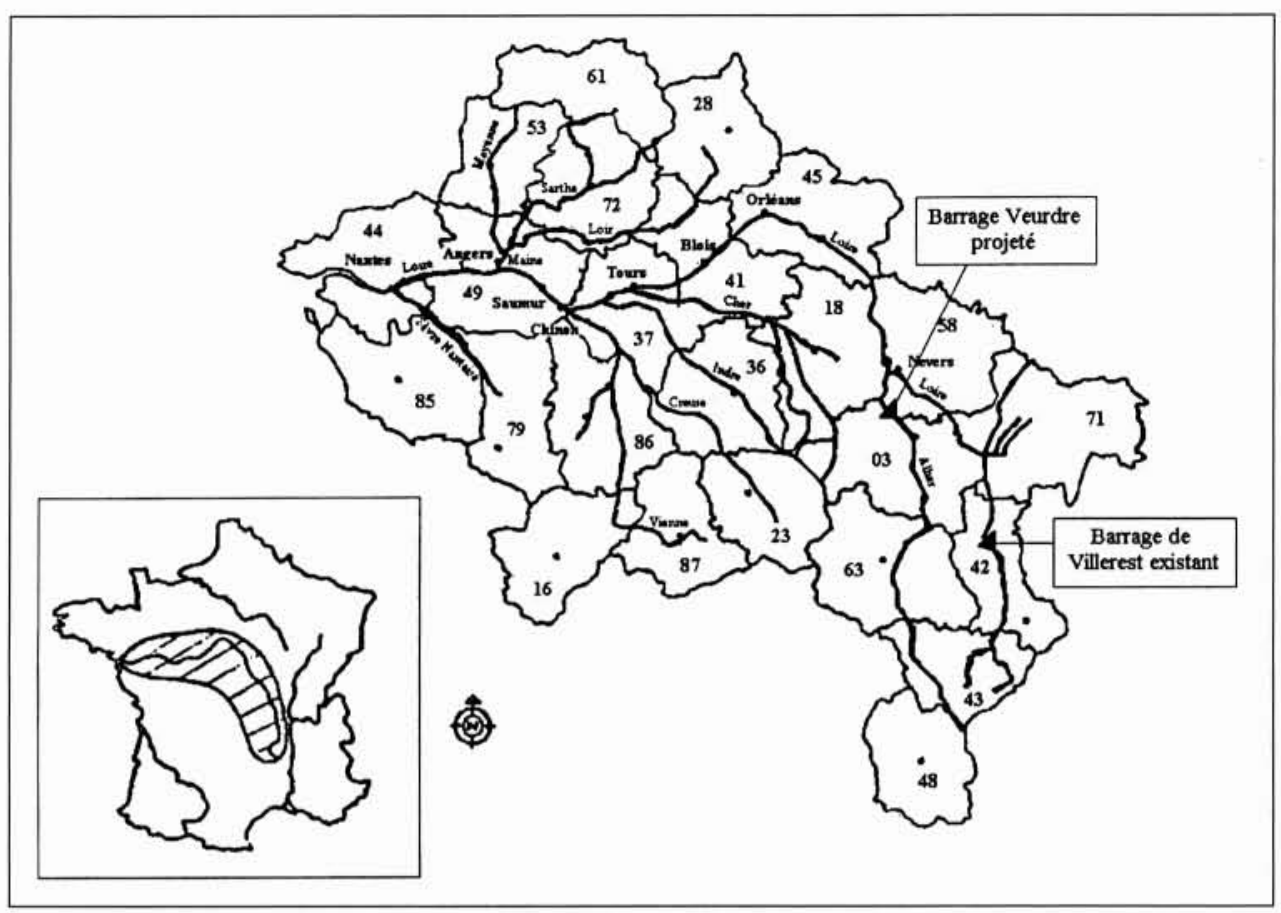

3. Localisation du secteur de Loire étudié et des barrages-réservoirs.

cations, est fortement non linéaire, et sa structure assez complexe. De fait, il n'est pas envisageable d'estimer les incertitudes analytiquement. Elles sont donc déterminées par l'intermédiaire de simulations de type Monte-Carlo : les données d'entrée et les paramètres du modèle sont considérés comme des variables aléatoires. L'écart-type des distributions utilisées se situe selon les paramètres entre 20 et $30 \%$ de la valeur moyenne. Il a été choisi de manière empirique, sur la base de références bibliographiques ou par calage dans le cas des paramètres caractérisant l'effet des barrages.

La loi de probabilité du coût des dommages est établie à partir de simulations répétées du modèle avec des valeurs de paramètres tirées au hasard. 500 simulations se sont avérées nécessaires pour établir cette loi de probabilité.

\section{IV - PRÉSENTATION DE L'ÉTUDE DE CAS}

L'application du modèle CIFLUPEDE, sur laquelle portent les résultats qui sont présentés par la suite, a consisté à évaluer la réduction des dommages sur une partie du cours de la Loire du fait de la réalisation d'un nouveau barrage (barrage Veurdre). Ce barrage est situé sur l'Allier, affluent de la Loire. Il est important de préciser que cet article vise, à partir d'une étude de cas réaliste, à illustrer les mécanismes de l'évaluation économique des dommages dus aux inondations et donner des ordres de grandeur des incertitudes attachées aux valeurs estimées. Il ne s'agit pas de se prononcer sur l'opportunité économique de la construction du barrage Veurdre qui fait actuellement l'objet d'études menées en particulier par l'équipe pluridisciplinaire Plan-Loire Grandeur Nature. Notons cependant qu'à notre connaissance les résultats des études économiques actuellement en cours sont tout à fait cohérents avec les valeurs présentées plus loin.

Le tronçon de 404 kilomètres étudié est situé entre les confluents de la Loire avec l'Allier en amont, et avec la Maine en aval (cf. Figure 3). Il est représenté dans le modèle par 8 biefs et 86 secteurs. Les principales agglomérations traversées par la Loire sur ce tronçon sont Orléans, Tour et Blois.
Deux effets des barrages-réservoirs ont été pris en compte dans le modèle.

- Une réduction du débit de pointe de crue pour une période de retour donnée. Cette réduction est d'autant moins notable que l'on progresse vers l'aval, et que les débits des affluents non contrôlés viennent grossir la crue. Cette réduction dépend aussi des apports de ces affluents : la relation entre le débit "naturel" et le débit de pointe de crue influencé par le barrage n'est donc pas bi-univoque. Pour chacun des biefs de l'étude une relation entre débit influencé et débit naturel a été calée à partir de données mesurées en crues et de données de débits influencés reconstituées par simulation hydraulique. Ce calage a aussi permis d'estimer l'incertitude liée à cette relation.

- Une modification des formes des hydrogrammes qui se traduit suivant les cas par une augmentation ou une diminution de la durée de submersion pour une crue donnée dépendant des débits naturels et écrêtés et du seuil de submersion. Une comparaison avec le modèle hydraulique a montré que le rapport des durées de submersion en situation naturelle et influencée était en moyenne inversement proportionnel au rapport des débits de pointe de crue dans le cas des biefs de cours d'eau non protégés par des levées.

\section{V — RÉSULTATS}

\subsection{Coûts des dommages en situation non influen- cée par les barrages}

Les résultats de calcul du coût des dommages potentiels d'inondations sur la Loire moyenne sont récapitulés dans le tableau 1.

Notons que pour ces premières simulations, la cohérence régionale ou locale de certaines sources d'incertitudes a été considérée : les incertitudes sur l'aléa hydrologique par exemple s'appliquent à l'ensemble des secteurs d'un même bief. Un seul tirage aléatoire par bief est donc effectué à chaque itération de la procédure Monte-Carlo. 


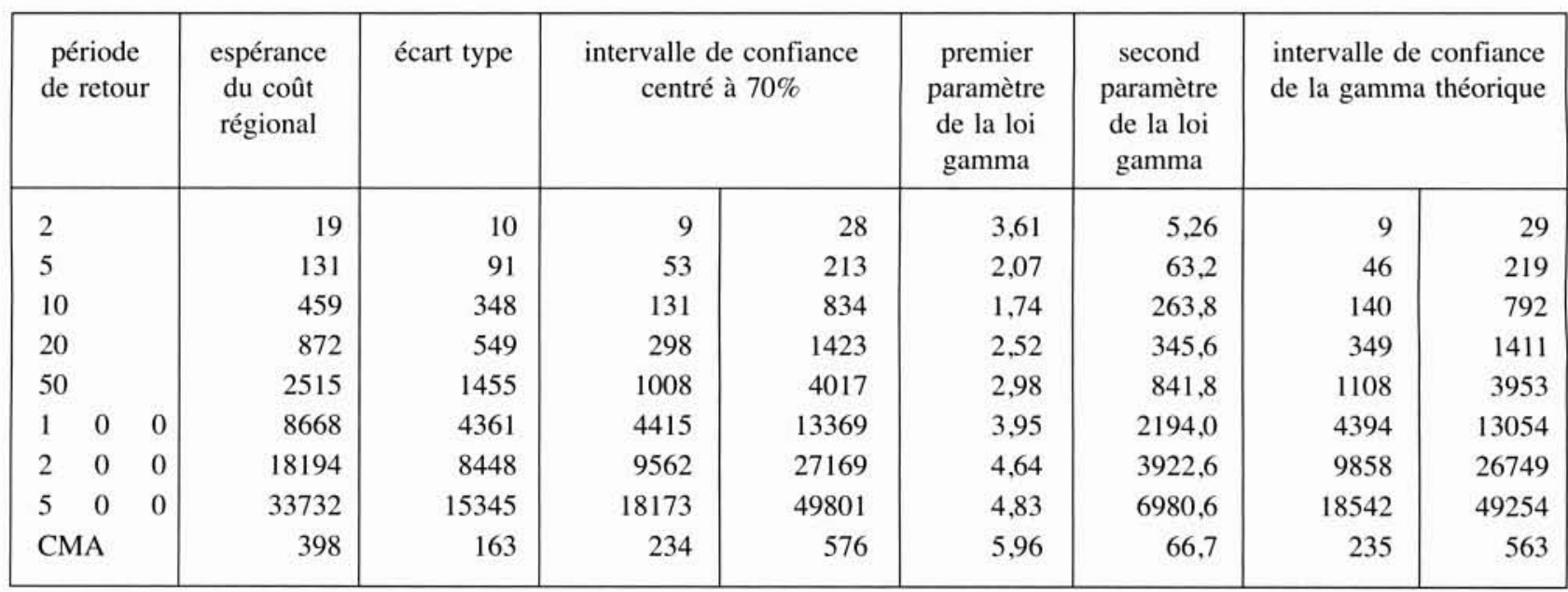

Tableau 1: Coût des dommages exprimé en millions de francs au troisième trimestre 1991 pour la Loire centrale, 500 simulations. Comparaison des distributions obtenues avec des distributions de type gamma. (CMA : Coût moyen annuel).

La prise en compte de la cohérence spatiale des sources d'incertitudes réduit notablement la dispersion des résultats de simulation (réduction de moitié de l'écart-type des distributions de coûts en moyenne). Ce résultat peut paraître contre-intuitif. En effet, on aurait pu s'attendre à ce que la réduction du nombre de tirages de variables aléatoires qu'implique le respect de la cohérence spatiale des incertitudes tende au contraire à augmenter la variance des résultats de simulation. Cela aurait probablement été le cas si la relation entre la période de retour de la crue et le coût des dommages avait été homogène sur l'ensemble des secteurs. Cette première remarque illustre combien les résultats que nous obtiendrons et les conclusions que nous pourrons en tirer peuvent dépendre des particularités du tronçon de vallée étudié, et de la manière dont elles sont retranscrites dans le modèle de calcul.

Remarquons aussi que le coût global des dommages pour la Loire moyenne a été obtenu par sommation des coûts calculés sur chacun des tronçons et des secteurs. Cette opération est licite dans le cas du coût moyen annuel. En revanche, elle l'est beaucoup moins dans le cas de crues de période de retour donnée. Rien n'assure en effet, compte tenu de la composition des crues de la Loire et de ses affluents, et de l'hétérogénéité de l'occupation des sols sur le bassin, que le coût des dommages de période de retour $\mathrm{T}$ corresponde à la somme des coûts de dommages de même période de retour sur chaque secteur. Le coût moyen annuel est la seule estimation pertinente à l'échelle régionale.

Les distributions de dommages obtenues sont assez bien représentées par des lois de probabilité de type Gamma (ie. les distributions peuvent être décrites par leur moyenne et leur variance), comme en témoignent la figure 4 et les calculs des intervalles de confiance théoriques du tableau 1.

La valeur moyenne du CMA calculée, environ 400 millions de francs, est à comparer à 1,5 milliard de francs : moyenne annuelle des dommages liés aux inondations en France depuis 1982 (date de promulgation de la loi d'indemnisation des victimes de catastrophes naturelles). Elle souligne l'importance des enjeux exposés aux inondations dans la vallée de la Loire, où la conscience collective du risque a pu s'estomper, la dernière grande crue datant de 1866

Notons enfin la forte dispersion des estimations de coût des dommages résultant de la prise en compte des incerti-

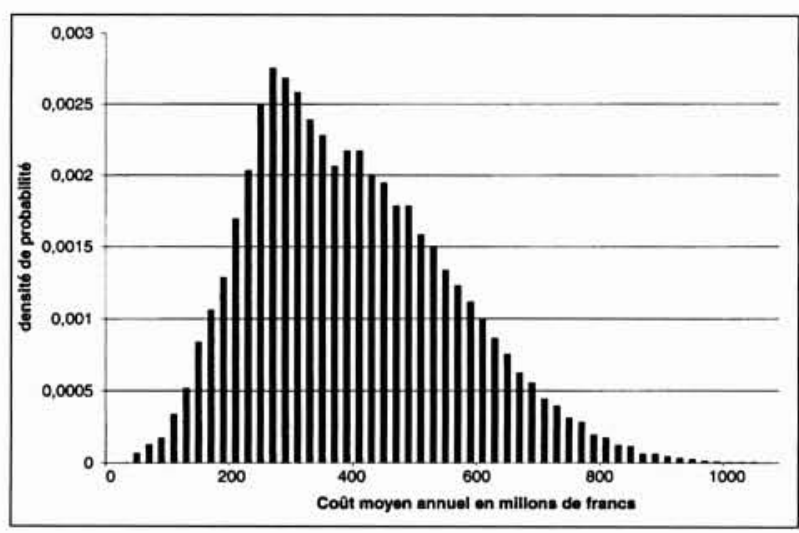

4. Distributions du coût moyen annuel pour la Loire moyenne.

tudes sur les données utilisées dans les calculs. Le rapport entre les bornes de l'intervalle de confiance centré à $70 \%$ est de l'ordre de 3 à 4 . Nous avons ici la confirmation que les coûts calculés doivent être considérés comme des ordres de grandeur : le coût moyen annuel des dommages liés aux crues sur la Loire moyenne, du moins ceux qui ont fait l'objet d'une évaluation économique, est de plusieurs centaines de millions de francs.

\subsection{Influence des barrages-réservoirs}

La réduction du coût moyen annuel des dommages est le critère que nous avons retenu pour juger de l'influence de l'aménagement existant (barrage de Villerest) et de l'aménagement envisagé (barrage du Veurdre). Le calcul a porté directement sur la réduction du coût des dommages. A chaque itération de Monte-Carlo les mêmes valeurs de paramètres du modèle et de données d'entrée sont appliquées à la situation de référence et à la situation aménagée, mis à part bien entendu les paramètres concernant l'influence des barrages.

L'effet des barrages se révèle être assez variable suivant les biefs et les périodes de retour considérés. Tout comme l'influence sur les débits de crue, l'influence des barrages sur le coût des dommages diminue de l'amont vers l'aval.

La combinaison des deux barrages permet de réduire de près 


\begin{tabular}{|l|c|c|c|c|c|}
\hline Comparaison des situations & $\begin{array}{c}\text { Espérance } \\
\text { de la } \\
\text { variation } \\
\text { de CMA }\end{array}$ & écart-type & $\begin{array}{c}\text { premier } \\
\text { paramètre } \\
\text { de la loi } \\
\text { gamma }\end{array}$ & $\begin{array}{c}\text { second } \\
\text { paramètre } \\
\text { de la loi } \\
\text { gamma }\end{array}$ & $\begin{array}{c}\text { Valeur } \\
\text { du premier } \\
\text { décile }\end{array}$ \\
\hline cas 1 : Etat naturel - barrage Villerest seul & 68 & 84 & 0,65 & 104 & 2,6 \\
\hline cas 2: Etat naturel - barrages Villerest et Veurdre & 152 & 164 & 0,85 & 177 & 11,4 \\
\hline $\begin{array}{c}\text { cas 3: barrage Villerest seul - barrages Villerest } \\
\text { et Veurdre }\end{array}$ & 86 & 96 & 0,80 & 107 & 5,7 \\
\hline
\end{tabular}

Tableau 2: Estimation des variations de coût moyen annuel (en millions de francs au troisième trimestre 1991) des dommages dues à l'aménagement des barrages-réservoirs.

de $40 \%$ l'espérance du coût moyen annuel des dommages sur la Loire moyenne par rapport à l'état naturel (cf. Tableau 2). Cette réduction n'atteint pas $20 \%$ dans le cas du seul barrage de Villerest. L'effet du barrage du Veurdre est donc notable.

Cependant, on aurait pu s'attendre à ce que la dispersion des estimations directes de réduction de coûts de dommages soit plus faible que la dispersion des estimations de coûts présentées dans la partie précédente. Il n'en est rien. Le coefficient de variation des distributions (écart-type divisé par la moyenne) est du même ordre de grandeur que celui des distributions de coût des dommages.

Notons que dans le cas où les coûts de construction et d'entretien du barrage Veurdre seraient à justifier au regard des dommages qu'il permet d'éviter - le barrage n'est réalisé que dans la mesure où l'on peut affirmer, avec un certain niveau de confiance, que ces coûts sont inférieurs aux montants des dommages évités - ces incertitudes sont susceptibles de modifier notablement la décision. En effet, en supposant que la distribution de réduction de CMA suit une loi gamma, $10 \%$ des valeurs calculées dans le cas numéro 3 (cf. Tableau 2) sont inférieures à 5,7 millions de francs.

L'estimation de la réduction du CMA déterministe (sans prise en compte des incertitudes) due à l'aménagement du barrage Veurdre par rapport au seul barrage Villerest permettrait de justifier un ouvrage dont le coût annuel d'amortissement et d'entretien s'élève à 86 millions de francs. La prise en compte des incertitudes d'estimation ne permet plus de justifier, avec un niveau de confiance de $90 \%$, que d'un ouvrage d'un coût de 5,7 millions, soit plus de dix fois moins.

\section{VI $\square$ DISCUSSION}

Dans le cas où les estimations de coûts de dommages sont utilisées dans le cadre d'une décision d'aménagement, les incertitudes peuvent jouer un rôle important comme nous venons de le voir. Il nous a semblé intéressant de déterminer quelle était la sensibilité des résultats de simulation aux différentes sources d'incertitudes, afin d'identifier les sources majeures sur lesquelles devront porter les efforts d'approfondissement de la collecte d'informations.

Les calculs de réduction de coût de dommages ont été effectués en éliminant successivement chaque source d'incertitudes, celles-ci ayant été regroupées en 12 catégories. Il apparaît que les sources d'incertitudes concernant l'aléa hydrologique contribuent en grande partie à la dispersion des résultats de réduction de CMA liée à l'effet du barrage Veurdre (cf : Figure 5). L'élimination de cette source permet de réduire de près de $60 \%$ l'écart-type des valeurs de

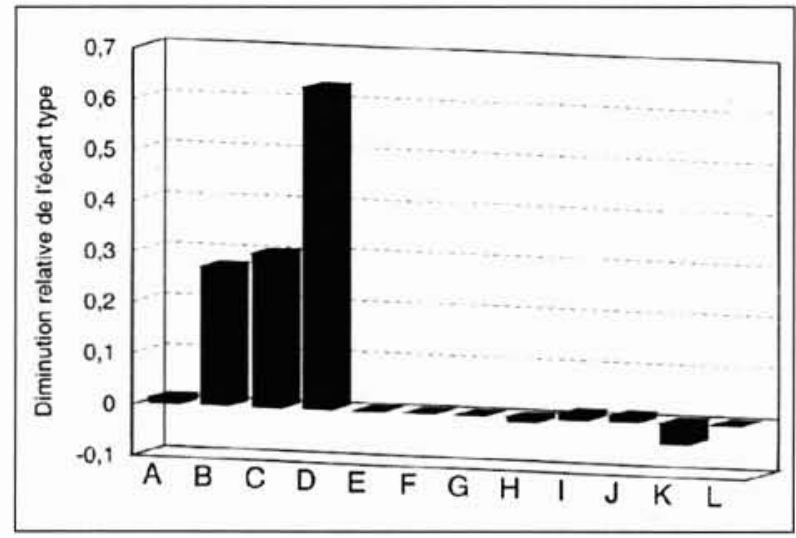

5. Diminution relative de l'écart-type de la distribution de la réduction du CMA liée à l'effet du barrage Veurdre par rapport à la simulation avec prise en compte de toutes les sources d'incertitude.

Les catégories de sources d'incertitudes testées sont les suivantes (A : effets des barrages, B : loi de probabilité des débits de pointe par bief , C : courbe de tarage, D : ensemble des incertitudes sur l'aléa hydrologique (T-Q-H), $\mathrm{E}$ : seuils de rupture de digue, $\mathrm{F}$ : fonctions de coût élémentaires, $\mathrm{G}$ : répartition des hauteurs et durées de submersion, $\mathrm{H}$ : niveau des planchers des habitations, I : loi hauteur - surface submergée par bief, J : occupation des sols, K : seuils de débordement des rives, L : seuil de déversement par-dessus les digues).

CMA calculées. On constate par ailleurs que la dispersion des résultats est pratiquement insensible à l'élimination des autres sources d'incertitudes. Dans certains cas elle aurait même contribué à augmenter l'écart-type des distributions de CMA. Ces résultats concordent avec ce qui a pu être obtenu lors de travaux précédents avec le modèle CIFLUPEDE [4].

Ils suggèrent que dans le cas de la Loire et du modèle CIFLUPEDE la collecte de données complémentaires afin de réduire l'une ou l'autre des sources d'incertitude concernant l'occupation des sols, l'hydraulique ou encore les données économiques ne permettrait pas d'augmenter notablement la précision des résultats obtenus. Ces résultats ne permettent en revanche pas de se prononcer sur l'effet de la réduction conjointe de plusieurs de ces sources. Par ailleurs, les résultats présentés plus haut révèlent l'importance des incertitudes liées à l'aléa hydrologique, dont une partie au moins est relativement irréductible en l'état actuel, la précision sur les lois de probabilité des débits de pointe de crue ne pouvant augmenter qu'avec la multiplication des données mesurées et donc avec le temps. 


\section{VII — CONCLUSION}

Il existe actuellement peu d'autres exemples d'estimation du coût des dommages liés aux inondations tenant compte des incertitudes qui permettraient de se prononcer sur la généralité des conclusions que l'on peut tirer des résultats présentés plus haut. Nous avons pu relever un certain nombre d'indices laissant supposer que le poids des différentes sources d'incertitude dans la dispersion des résultats, et leurs interactions risquent d'être largement dépendants de la structure du modèle utilisé, du type de représentation des phénomènes, du choix des valeurs des écarts-types et des distributions des paramètres du modèle. Ce choix, rappelons-le, a été plus ou moins empirique. La sensibilité des résultats à la valeur des enjeux exposés ou aux fonctions d'endommagement aurait certainement été plus grande si la zone étudiée avait contenu un ou plusieurs établissements particuliers représentant une part importante du coût total des dommages ?

Il est cependant possible de tirer quelques enseignements sur l'utilité de l'estimation du coût des dommages liés aux inondations pour l'évaluation de projets de prévention et sur les limites de ce type d'approche.

- Le calcul du coût des dommages vise bien évidemment à fournir des ordres de grandeur. Le coût des dommages évités ne peut être le seul, ni même le premier critère de choix pour la réalisation d'un aménagement. Il faudrait pour le moins être en mesure de répertorier de manière exhaustive les dommages potentiels. Par ailleurs, on raisonne le plus souvent en situation actuelle d'urbanisation, et parfois en considérant des scénarios de développement. Il est cependant difficile de tenir compte, dans le cadre d'une évaluation économique, de l'éventuel impact de la réalisation d'ouvrages de protection sur l'urbanisation future et donc sur la vulnérabilité des secteurs inondables.

- Compte tenu des interactions complexes entre les différentes sources d'incertitudes dans le calcul des dommages, l'approfondissement des connaissances concernant l'une des données ou l'un des paramètres utilisés pour le calcul (modèle d'occupation des sols plus détaillé, valeur des enjeux exposés mieux connues par exemple) ne se traduira pas nécessairement par une plus grande précision des valeurs calculées. Cela vaudra probablement quel que soit le type de modèle utilisé. Nous illustrons ici un principe bien connu des économistes qu'Alfred Sauvy exposait avec humour : "Plus nous comptons, plus nous comptons mal, puisque nous ne comptons pas tout".

- Les incertitudes, irréductibles par nature, concernant la fréquence des crues fixent en quelque sorte le niveau de précision du calcul du coût des dommages et en particulier du CMA qui ne pourra être dépassé. Elles peuvent rapidement devenir le facteur limitant la précision des évaluations économiques.

\section{Références}

[1] BCEOM (1967), - Essai sur le coût économique des crues. Ministère de l'équipement et du logement, 3 volumes (104 pages, 193 pages, 186 pages).

[2] BCEOM (1973). - Evaluation des dommages de crue dans la vallée du Cher. Agence financière de bassin Loire-Bretagne. 133 pages.

[3] BCEOM (1990). - Dommages de crues dans le bassin de la Moselle. Ministère de l'Environnement. 251 pages.

[4] TORTEROTOT J-P. (1993), - Le coût des dommages dus aux inondations : estimation et analyse des incertitudes. Thèse de doctorat de l'Ecole nationale des ponts et chaussées, Paris, 283 pages, annexes.

[5] ARNal. C., Masure P. (1996). - Approche intégrée des risques dus aux aléas naturels. Evaluation de l'impact économique et financier d'une catastrophe naturelle : le cas de Nìmes. BRGM, Ministère de l'Environnement, 69 pages, annexes.

[6] BCEOM et SAGERY (1997). - Etude économique des crues de l'Orne. Syndicat mixte de lutte contre les inondations dans la vallée de l'Orne et son bassin versant. 42 pages et annexes.

[7] Devaux-Ros C. (1998). - Evaluation des enjeux et des dommages potentiels liés aux inondations de la Loire moyenne, méthodes et principaux résultats. Equipe pluridisciplinaire Plan Loire Grandeur Nature. Agence de l'eau Loire-Bretagne, 46 pages et annexes.

[8] HYDRATEC, SIEE, Territoires Conseils (1998).- Evaluation des dommages liés aux crues en région Ile-de-France, rapport de synthèse, Les grands lacs de Seine. 107 pages et annexes.

[9] Laglaine V., Hubert G. (1995). - Assessment of flood damage cost reductions due to tructural river flood control projects and analysis of associated uncertainties. Workshop Euroflood 2, Londres. 28 pages.

[10] Desgranges P. (1998). La vie des entreprises : évaluation des dommages indirects aux activités. La Houille Blanche, 346 (1), p. 72-75.

[11] GAzULL. L. (1998). Elaboration d'un outil dynamique d'évaluation des dommages des crues sur l'ensemble de la région Ile-de-France. La Houille Blanche, 346 (1), p. 76-80.

[12] RizzoL J-P. (1997). Le risque de crue en région parisienne : démarche et projets de l'institution. . La Houille Blanche, 345 (8), p. 71-76.

[13] BCEOM (1970). - Recherche méthodologique sur l'évaluation des coûts unitaires des dommages causés par les crues. Ministère de l'Equipement et du Logement. 212 pages.

[14] SAGERI (1988). - Evaluation de la vulnérabilité. Ministère chargé de I'Environnement, DEPPR, DRM. 38 pages, annexes.

[15] Deleuze C., Fotre C., Nut 1., Pierrot F. (1991).- Evaluation des fonctions de coûts économiques des dommages aux cultures dus aux inondations. Ecole nationale du génie rural des eaux et des forêts, Paris, 50 pages.

[16] Parker D. J., Green C.H., Thompson P.M. (1987).- Urban flood protection benefits, a project appraisal guide, Gower Technical Press, Aldershot. 150 pages, annexes.

[17] Penning-Rowsell. E. C.. Chatterton J. B. (1977)- The benefits of flood alleviation, a manual of assessment techniques, Gower Technical Press, Aldershot. 131 pages, annexes.

\section{Remerciements}

Les travaux de recherches dont quelques résultats sont présentés dans cet article ont bénéficié du soutien financier du ministère de l'Environnement de 1988 à 1993 et de la Communauté économique européenne de 1993 à 1996 au travers des programmes de recherche Euroflood I et Euroflood II. 